\title{
Hydrophobic nanoconfinement suppresses fluctuations in supercooled water
}

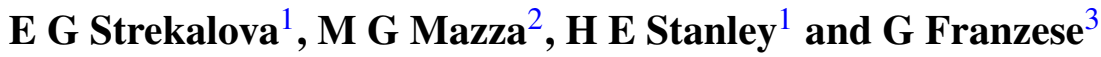 \\ ${ }^{1}$ Center for Polymer Studies and Department of Physics, Boston University, Boston, MA 02215, USA \\ ${ }^{2}$ Stranski-Laboratorium für Physikalische und Theoretische Chemie, Technische Universität Berlin, \\ Straße des 17. Juni 135, D-10623 Berlin, Germany \\ ${ }^{3}$ Departament de Física Fonamental, Universitat de Barcelona, Diagonal 645, E-08028 Barcelona, Spain \\ E-mail: elena@buphy.bu.edu
}

Received 18 May 2011, in final form 13 December 2011

Published 25 January 2012

Online at stacks.iop.org/JPhysCM/24/064111

\begin{abstract}
We perform very efficient Monte Carlo simulations to study the phase diagram of a water monolayer confined in a fixed disordered matrix of hydrophobic nanoparticles between two hydrophobic plates. We consider different hydrophobic nanoparticle concentrations $c$. We adopt a coarse-grained model of water that, for $c=0$, displays a first-order liquid-liquid phase transition (LLPT) line with negative slope in the pressure-temperature $(P-T)$ plane, ending in a liquid-liquid critical point at about $174 \mathrm{~K}$ and $0.13 \mathrm{GPa}$. We show that upon increase of $c$ the liquid-gas spinodal and the temperature of the maximum density line are shifted with respect to the $c=0$ case. We also find dramatic changes in the region around the LLPT. In particular, we observe a substantial (more than 90\%) decrease of isothermal compressibility, thermal expansion coefficient and constant-pressure specific heat upon increasing $c$, consistent with recent experiments. Moreover, we find that a hydrophobic nanoparticle concentration as small as $c=2.4 \%$ is enough to destroy the LLPT for $P \geq 0.16 \mathrm{GPa}$. The fluctuations of volume apparently diverge at $P \approx 0.16 \mathrm{GPa}$, suggesting that the LLPT line ends in an LL critical point at $0.16 \mathrm{GPa}$. Therefore, nanoconfinement reduces the range of $P-T$ where the LLPT is observable. By increasing the hydrophobic nanoparticle concentration $c$, the LLPT becomes weaker and its $P-T$ range smaller. The model allows us to explain these phenomena in terms of a proliferation of interfaces among domains with different local order, promoted by the hydrophobic effect of the water-hydrophobic-nanoparticle interfaces.
\end{abstract}

(Some figures may appear in colour only in the online journal)

\section{Introduction}

Water is frequently found in nature in its supercooled state. This fact has stimulated intense experimental and theoretical research [1-10]. In the deeply supercooled region, however, the direct observation of bulk water is extremely difficult due to the inevitable crystallization that occurs. One way to overcome this experimental difficulty is to confine water to nanometric distances. Nanoconfined water remains liquid down to very low temperatures [3], opening the possibility of exploring the properties of confined water in a temperature range that is inaccessible when the subject is bulk liquid water. A number of important technological and biological applications depend on understanding the phase diagram of water [4-15]. For example, hydrophobic and hydrophilic interactions are important driving forces for the self-assembling of micelles or membranes and are fundamental for protein folding. Water is enclosed in cell membranes and is a typical solvent for proteins [16]. The study of water confined in carbon nanotubes is opening new technological perspectives [17], and thus there has been increasing interest in studying confined water in various geometries, such as nanopores or nanotubes, protein hydration water and intracellular water [18-26].

One very peculiar property of supercooled water is the phenomenon of polyamorphism, i.e. the presence of two or more liquid or glassy states, which was first observed in glassy water [27-29]. There are two different forms of 
amorphous glassy water: a low density amorphous form (LDA) and a high density amorphous form (HDA) that are separated by a first-order phase transition line. Consequently, polyamorphism for liquid water with a low density liquid (LDL) and a high density liquid (HDL) was proposed [30, 31] triggering experimental investigations [32,33]. A number of current theories and models predict a first-order liquid-liquid phase transition (LLPT) for bulk water between LDL and HDL below the homogeneous nucleation temperature $T_{\mathrm{H}}^{\text {bulk }}$, where bulk water freezes spontaneously [2, 30, 34-45]. Recently, Limmer and Chandler performed free energy calculations for the ST2 water model, concluding that there is no evidence for the LLPT [46]. However, more recent calculations reach a different conclusion supporting the existence of the LLPT [47-50].

Recent studies on water in various confined geometriesin which water crystallization is suppressed down to very low temperatures-have investigated the region in the pressure-temperature $(P-T)$ phase diagram where a first-order LLPT is predicted. However, no definitive answer has been given regarding the thermodynamic implications of confinement and its relevance to the phase diagram of bulk water. Water differs from most liquids in that there is an important thermodynamic locus in the $P-T$ phase diagram - the line where, at a given $P$, the $T$ dependence of the density exhibits a maximum, commonly referred to as the temperature of maximum density (TMD). A shift to lower $T$ of the TMD line, and its curvature modification, have been reported for confined water, suggesting important changes in the phase diagram deep in the supercooled region [51, 52]. In general, experiments and simulations [53-55] show that LLPTs in a confined space can differ from those in bulk. Therefore, there are aspects of the thermodynamics of confined water that remain open to debate $[56,57]$.

In computer simulations, one possible approach to these studies is to develop atomistic models of water utilizing a geometry such as slits [58-62] or a disordered matrix of discs or spheres [51,52]. The main difficulty in this approach is that these simulations are time-consuming and are usually limited to only a few hundred water molecules.

Another possible approach is to consider coarse-grained models of water. Coarse graining can be done at different levels, i.e. at the level of a single water molecule [34, 63, 64] or a few molecules [65], depending on the properties being studied. Here we present results for a water model that is coarse-grained at the single-molecule level. Molecular details are sacrificed for the benefit of computational speed, which allows more extensive studies, and theoretical simplicity, which allows analytical calculations. Here we ask whether the confinement in a fixed matrix of hydrophobic nanoparticles changes the thermodynamics of a water monolayer at temperatures below $T_{\mathrm{H}}^{\text {bulk }}$.

Using Monte Carlo simulations, our results for a coarse-grained model of a water monolayer [66] show that even a small presence of hydrophobic nanoparticles can drastically suppress thermodynamic fluctuations and wash out the coexistence of the two types of liquid supercooled water at high pressures.
This paper is organized as following. Sections $2-4$ outline details of the model and Monte Carlo simulations. Section 5 presents the results. Discussion and conclusions are given in section 6 .

\section{Coarse-grained model of water monolayer}

We consider a coarse-grained model for water in two dimensions confined between two smooth hydrophobic plates, whose interaction with water is purely repulsive and represented by a steric hard-core exclusion. It has been previously shown that, when the distance between the plates is approximately $0.5 \mathrm{~nm}$, the monolayer of water remains in its liquid phase, while the properties of the confined water are only weakly dependent on the details of the confining potential between smooth walls $[67,68]$. We partition a water monolayer of thickness $h \simeq 0.5 \mathrm{~nm}$ and volume $\mathscr{V}$ into $\mathscr{N}$ square cells of equal length $\sqrt{\mathscr{V} / \mathscr{N} h}$. We coarse-grain over the position orthogonal to the walls and consider only the projection of water molecules in a plane parallel to the wall. We further coarse-grain the detailed position of each molecule, discretizing it to the resolution given by our square partition of the parallel plane, assigning to each cell an occupancy variable $n_{i}=1$ if the cell is occupied by a water molecule or $n_{i}=0$ if it is occupied by a hydrophobic nanoparticle. We consider the case in which there are no empty cells in the system and hydrophobic nanoparticles can occupy more than one cell, depending on their size. Hydrophobic nanoparticles are discs of radius $R$ and are approximated by a set of cells that fall within the $\pi R^{2}$ area centered on each hydrophobic nanoparticle (figure 1(a)). Hydrophobic nanoparticles are randomly distributed and form a fixed matrix that mimics a porous system or a rough atomic interface (figure 1(b)). $N \leq \mathscr{N}$ denotes the total number of cells occupied by water molecules and $V \leq \mathscr{V}$ denotes their total volume.

In order to implement a constant $P$ ensemble we include volume $V$ fluctuations in the model by allowing a global rescaling of the cell length $\sqrt{\mathscr{V} / \mathscr{N} h}$. The cell length coincides with the distance among nearestneighbor molecules. Therefore, the volume fluctuations induce fluctuations of $r_{i j}$ : (i) the distance between any two water molecules $i$ and $j$ and (ii) the distance between any water molecule $i$ and any cell $j$ occupied by the nanoparticle. Since a continuous range of possible volume fluctuations is allowed, the distances change as continuous variables, despite the discrete lattice partition of the space. As a result, the fluctuations of the cell size and therefore the fluctuations of the volume occupied by a nanoparticle are a consequence of the change of the coarse-graining scale. Therefore, it does not imply that the nanoparticle volume is increasing or decreasing, but that the coarse-graining length scale undergoes (small) fluctuations.

The system is described by the Hamiltonian [34-40]

$$
\mathscr{H} \equiv \sum_{i j} U\left(r_{i j}\right)-J N_{\mathrm{HB}}-J_{\sigma} \sum_{i} n_{i} \sum_{(k, \ell)_{i}} \delta_{\sigma_{i k}, \sigma_{i \ell}} .
$$

The first term, $U\left(r_{i j}\right) \equiv U_{\mathrm{w}}\left(r_{i j}\right)+U_{\mathrm{h}}\left(r_{i j}\right)$, is given by the sum of the isotropic part of the water-water interaction $U_{\mathrm{w}}\left(r_{i j}\right)$ 

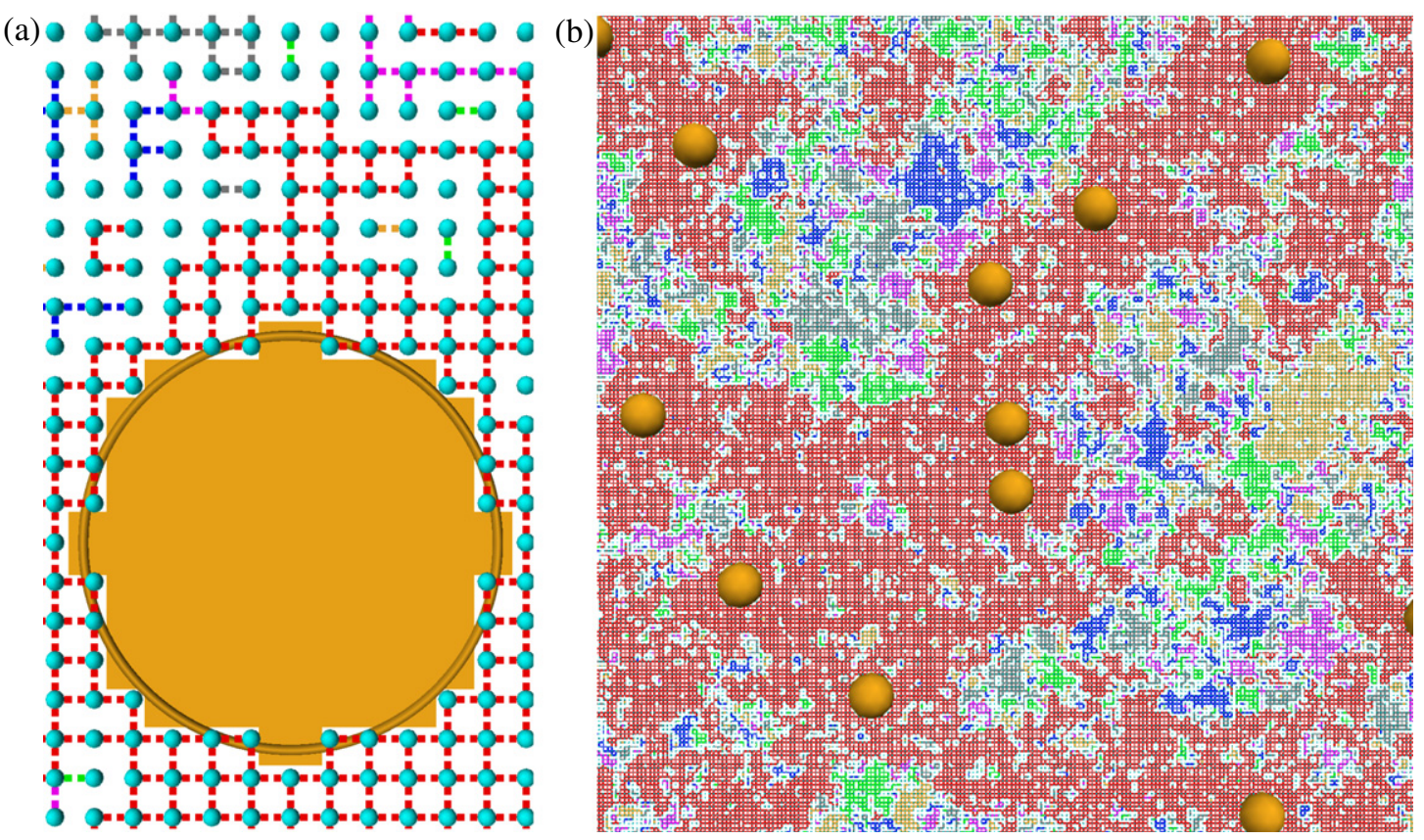

Figure 1. (a) Snapshot magnified around the region occupied by the hydrophobic nanoparticle (golden irregular polygon). The size of the hydrophobic nanoparticle is controlled by the number of cells it can occupy, and its shape is approximated by a disc composed of cells that fall within a radius $R$. Here the radius of the hydrophobic nanoparticle is $R=1.6 \mathrm{~nm}$. Small cyan spheres are water cells with four bond indices (small squares) with six possible colors, corresponding to the $q=6$ possible values of the bonding variables. Only the bond indices that participate in the formation of the hydrogen bond are shown here. (b) Snapshot of the monolayer with $2.4 \%$ of its volume occupied by hydrophobic nanoparticles (large golden spheres) with $R=1.6 \mathrm{~nm}$. Hydrophobic nanoparticles are randomly placed and form a fixed matrix. Hydrogen bonds are visualized by different color lines between water cells (small cyan spheres), depending on their relative state.

and the water-nanoparticle interaction $U_{\mathrm{h}}\left(r_{i j}\right)$. The two pair interactions are defined as

$$
\begin{array}{r}
U_{\mathrm{w}}(r) \equiv \begin{cases}\infty & \text { for } r<r_{0}, \\
\epsilon_{\mathrm{W}}\left[\left(\frac{r_{0}}{r}\right)^{12}-\left(\frac{r_{0}}{r}\right)^{6}\right] & \text { for } r \geq r_{0}, \\
0 & \text { for } r>r_{\mathrm{c}}\end{cases} \\
U_{\mathrm{h}}(r) \equiv \begin{cases}\infty & \text { for } r<r_{0}, \\
\epsilon_{\mathrm{h}}\left[\left(\frac{r_{0}}{r}\right)^{12}\right] & \text { for } r \geq r_{0}, \\
0 & \text { for } r>r_{\mathrm{c}}\end{cases}
\end{array}
$$

where $r_{0} \equiv 2.9 \AA$ is the water van der Waals diameter, $\epsilon_{\mathrm{w}} \equiv 5.8 \mathrm{~kJ} \mathrm{~mol}^{-1}$ is the water-water attraction energy, $r_{\mathrm{c}}=$ $\sqrt{\mathscr{V} / h} / 4$ is the cutoff distance. For the water-water case this term includes the short-range repulsion of the electron clouds and all the isotropic long-range attractive interactions, such as the weak instantaneous induced dipole-dipole (London) interactions between the electron clouds of different molecules and the stronger isotropic part of the hydrogen bond [69].

For the water-hydrophobic-nanoparticle case we assume that the interaction is purely repulsive, with $\epsilon_{\mathrm{h}} \equiv \epsilon_{\mathrm{w}} \sqrt{0.1}=$ $1.8 \mathrm{~kJ} \mathrm{~mol}^{-1}$ to soften the repulsive ramp. We observe here that a hydrophobic nanoparticle could also have a small attractive term in the water-hydrophobic-nanoparticle interaction, since dispersive van der Waals forces are always present. However, the repulsive interaction is typically one order of magnitude stronger than the attractive term [70].
Our neglect of the attractive water-nanoparticle interaction is, therefore, reasonable at first approximation and has been adopted by other authors [51].

The second term of equation (1) describes the strong directional component of the hydrogen bond (HB) due to the dipole-dipole interaction between the highly concentrated positive charge on each $\mathrm{H}$ and each of the two excess negative charges concentrated on the $\mathrm{O}$ of another water molecule. To account for the different bonding configurations of each water molecule, we assign to each cell $i$ four bond variables $\sigma_{i j}=$ $1, \ldots, q$ (one for each nearest-neighbor cell $j$ ), representing the orientation of molecule $i$ with respect to molecule $j$. A common assumption is that an $\mathrm{HB}$ breaks when $\widehat{\mathrm{OOH}}>30^{\circ}$. Therefore, only $1 / 6$ of the orientation range $\left[0^{\circ}, 360^{\circ}\right]$ in the $\mathrm{OH}-\mathrm{O}$ plane is associated with a bonded state. Hence, every molecule has $q^{4}=6^{4} \equiv 1296$ possible orientations and by considering the index $\sigma_{i j}$ we account for the entropy loss associated with the formation of an HB. We say that two molecules in nearest-neighbor (n.n.) cells form an HB only if they are correctly oriented, by definition, $\delta_{\sigma_{i j}, \sigma_{j i}} \equiv 1$ if $\sigma_{i j}=\sigma_{j i}, \delta_{\sigma_{i j}, \sigma_{j i}} \equiv 0$ otherwise. The total number of HBs is

$$
N_{\mathrm{HB}} \equiv \sum_{\langle i, j\rangle} n_{i} n_{j} \delta_{\sigma_{i j}, \sigma_{j i}},
$$

where $n_{i} \equiv 0$ for a nanoparticle and 1 for a water molecule. The notation $\langle i, j\rangle$ denotes that the sum is performed over n.n. water molecules $i$ and $j$, so that each water molecule can form up to four HBs. We consider a bond energy $J \equiv 2.9 \mathrm{~kJ} \mathrm{~mol}^{-1}$, 
as adopted in [38]. Since $\epsilon_{\mathrm{w}}=2 J$, the HBs are formed in large numbers only in the condensed liquid phase.

Hydrophobic nanoparticles are treated as a fixed random confining matrix and the bonding variable facing the nanoparticles cannot participate in any hydrogen bond, but contribute to the entropy of the system and are correlated to the other three bonding variables of the same water molecule.

Experiments have demonstrated that the formation of an HB leads to an open, locally tetrahedral, structure that induces an increase of volume per molecule within the second shell [71, 72]. We incorporate this effect by an enthalpy increase $P v_{\mathrm{HB}}$ for each $\mathrm{HB}$, where $P$ is pressure and $v_{\mathrm{HB}} / v_{0}=$ 0.5 is the average volume increase from high density ices VI and VIII to low density ice Ih, and $v_{0} \equiv h r_{0}^{2}$. Then the total water volume of the system is defined as

$$
V \equiv V_{0}+N_{\mathrm{HB}} v_{\mathrm{HB}}
$$

where $V_{0} \geq N v_{0}$ is a continuous variable that changes with pressure in such a way that $V$ follows the water equation of state [73]. Note that only the term $V_{0}$ of the fluctuating volume is considered for the calculation of distances $r$ appearing in equation (2) for the isotropic interaction $U(r)$, because the $\mathrm{HB}$ formation does not imply an increase of molecular distances, but only an increase of the local tetrahedral structure with the exclusion of interstitial water molecules between the first and the second shell. This exclusion induces the increase of volume per water molecule mimicked by equation (4).

The third term accounts for the experimental fact that at low $T$ the $\mathrm{O}-\mathrm{O}-\mathrm{O}$ angle distribution in water becomes sharper around the tetrahedral value [57], consistent with a cooperative behavior among bonds [37] as a consequence of the many-body interaction of water molecules. We model this interaction by including a coupling $J_{\sigma} \equiv 0.29 \mathrm{~kJ} \mathrm{~mol}^{-1}$ among the four bonding indices of the same water molecule in the third term of equation (1), where $(k, \ell)_{i}$ denotes each of the six different pairs of the four bond indices $\sigma_{i j}$ of a molecule $i$. Since $J_{\sigma}<J$, this term locally drives the HBs formed by a water molecule towards an ordered configuration, mimicking the many-body interaction that induces the locally ordered structure of the HBs.

\section{Hydrophobic interaction}

Confinement along one dimension inhibits the formation of 3D ice at $T<T_{\mathrm{H}}^{\text {bulk }}$ [62] and forces the water molecules to freeze into a lattice characterized by orientational disorder [62]. The characteristics of the lattice are closely related to those of the hydrophobic surface. For example, strong template effects are present for the graphene surface [74] while the absence of atomic characterization of the surface leads to a generic square arrangement [62]. In any case, the limited space left open to water induces the formation of an almost 2D structure where the translational dynamics at low $T$ is very limited, while the HB breakingand-formation dynamics is present also at low $T$ [62]. All of these features are reproduced in the coarse-grained model considered here [60]. Moreover, the possibility of exploring very low temperatures and very high pressures by means of this coarse-grained water model allows us to predict a phase diagram with a first-order LLPT, between an LDL and an HDL, starting at $P \simeq 0.2 \mathrm{GPa}$ for $T \rightarrow$ 0 and ending in a critical point at $T \simeq 174 \mathrm{~K}$ and $P \simeq$ $0.13 \mathrm{GPa}$ [34-40] (figure 6(a)). Furthermore, in the vicinity of the liquid-liquid critical point, the HB dynamics displays the largest heterogeneity, consistent with the presence of cooperative dynamics [60], and with experiments for a water monolayer hydrating hemoglobin [75].

In the case considered here, water is also confined by hydrophobic nanoparticles to the two directions parallel to the infinite flat surfaces. As shown in (2), the water-hydrophobicnanoparticle interaction is repulsive and leads to interesting physics at low $T$. In particular, it has been proposed that supercooled water forms highly structured regions in the hydration shell of nonpolar solutes [76], where the hydrogen bond network is weakened only when the size of the hydrophobic nanoparticles is above a characteristic value [77], calculated using free energy analysis to be $\approx 1 \mathrm{~nm}$ [78]. Moreover Muller showed that experimental results can be explained only by assuming enthalpic strengthening of the hydration HBs with a simultaneous entropy increase in the hydration shell [79].

In our model the restructuring effect of hydrophobic nanoparticles on water is incorporated by replacing the parameter $J$ and $J_{\sigma}$ in the hydration shell with $J^{\mathrm{h}}=1.30 J$ and $J_{\sigma}^{\mathrm{h}}=1.30 J_{\sigma}$, following Patel et al [80]. Because bonding indices facing the hydrophobic nanoparticle cannot form HBs, at intermediate $T$ they have a number of accessible states larger than those facing water molecules, inducing an increase of hydration entropy consistent with the description of the hydrophobic effect given by Muller [79] and subsequently modified by Lee and Graziano [81].

\section{Monte Carlo simulations}

We perform Monte Carlo (MC) simulations in two dimensions for constant $P, T$ and $N$. We use the protocol of heating the system starting from a completely ordered configuration. We simulate systems with $\mathscr{N} \leq 1.6 \times 10^{5}$ within a fixed matrix of hydrophobic nanoparticles of radius $R=1.6 \mathrm{~nm}$, with hydrophobic nanoparticle concentration $c \equiv(\mathscr{N}$ $N) / \mathscr{N}=2.4 \%$ and $25 \%$. We repeat the analysis for $R=$ $0.4 \mathrm{~nm}$ for a range of concentrations between $0.5 \%$ and $22.5 \%$ for a fixed system size of $\mathscr{N}=10^{4}$. We observe that for the two hydrophobic nanoparticle radii the effect is the same as long as the amount of hydrophobic interface in contact with water is the same. Hence, the concentration $c$ necessary to observe the same effect is larger for the smaller hydrophobic nanoparticles, the relevant factor being the amount of hydrophobic interface. We discuss our results in terms of $c$ and $R$.

In our simulations we update the variables $\sigma_{i j}$ using the Wolff cluster algorithm [73]. The algorithm is based on an exact mapping of the model studied here to a percolation problem, following the mapping rules described in $[82,83]$. The mapping is exact in this case because the system has no frustration. Bond indices within a water molecule belong to 


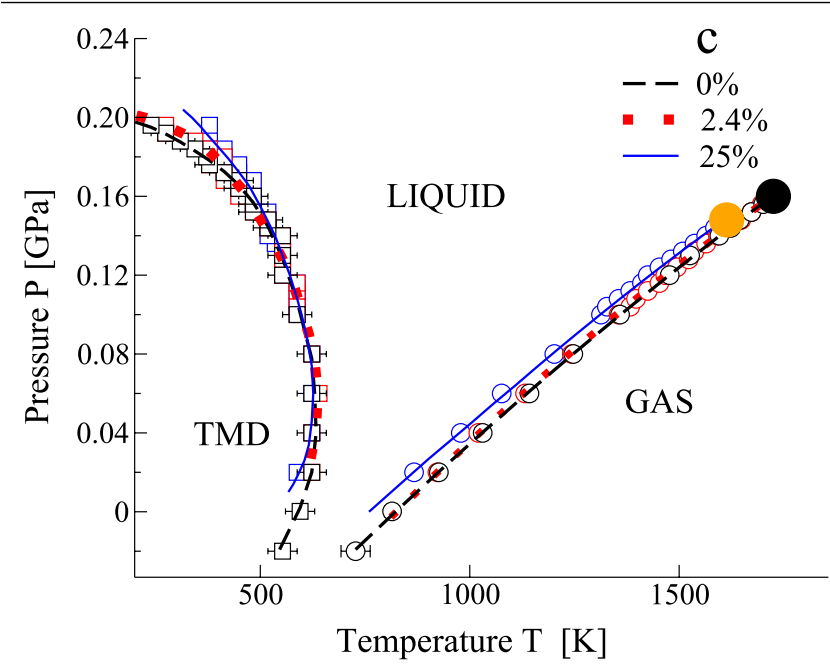

Figure 2. $P-T$ phase diagram for different hydrophobic nanoparticle concentrations $c$. Open circles mark liquid-to-gas spinodal line, while squares mark TMD line. In this and all other figures, where not shown, errors are smaller than the symbol size. Lines are guides for the eyes (dashed for $c=0$, dotted for $2.4 \%$, full for $25 \%$ ). The liquid-gas critical point (large full circle) is the same, within the error bar, for $c=0$ and $2.4 \%$, while it occurs at lower $P$ and lower $T$ for $c=25 \%$.

the same cluster with probability $p_{\text {same }} \equiv 1-\exp \left[-\beta J_{\sigma}\right]$, where $\beta \equiv\left(k_{\mathrm{B}} T\right)^{-1}$ and $k_{\mathrm{B}}$ is the Boltzmann constant. Bond indices of nearest-neighbor (n.n.) water molecules belong to the same cluster with probability $p_{\text {facing }} \equiv 1-\exp \left[-\beta J^{\prime}\right]$, where $J^{\prime} \equiv J-P v_{\mathrm{HB}}$ is the $\mathrm{HB}$ enthalpy due to the interaction energy and the HB volume increase. For the interfacial water molecules, we consider $J^{\mathrm{h}}$ and $J_{\sigma}^{\mathrm{h}}$ instead of $J$ and $J_{\sigma}$, respectively. We study pressures in the interval $0.02 \mathrm{GPa}$ $\leqslant P \leqslant 0.2 \mathrm{GPa}$ and we present a detailed scaling analysis for pressures $0.12,0.14,0.16$ and $0.18 \mathrm{GPa}$.

\section{Results}

We first considered how the confinement in a fixed hydrophobic matrix affects the thermodynamics of liquid water above the melting point. In figure 2 the $P-T$ phase diagram for different concentrations $c$ of hydrophobic nanoparticles demonstrates a shift to lower $T$ of the liquid-gas spinodal for $c>0$. For $c=25 \%$ the $T$ change from the $c=0$ case is about $55 \mathrm{~K}$ at $P=0.02 \mathrm{GPa}$ and about $35 \mathrm{~K}$ at $P=0.14 \mathrm{GPa}$. The shifts in the TMD for $c>0$ with respect to the $c=0$ case is reminiscent of results for other models of confined water [51, 62]. We find stronger changes for increasing $c$. The changes are not monotonic with pressure. For instance, for $c=25 \%$ the TMD exhibits a shift to lower $T$ of about $35 \mathrm{~K}$ at $P=0.02$ and $0.14 \mathrm{GPa}$, whereas at $P=0.19 \mathrm{GPa}$ the TMD increases by about $100 \mathrm{~K}$ with respect to the $c=0$ case (figure 2).

Relevant properties are associated with the fluctuations of thermodynamic quantities. For example, the fluctuations of an order parameter associated with a phase transition exhibit characteristic behavior when the phase transition occurs. Hence, their calculation allows us to locate the

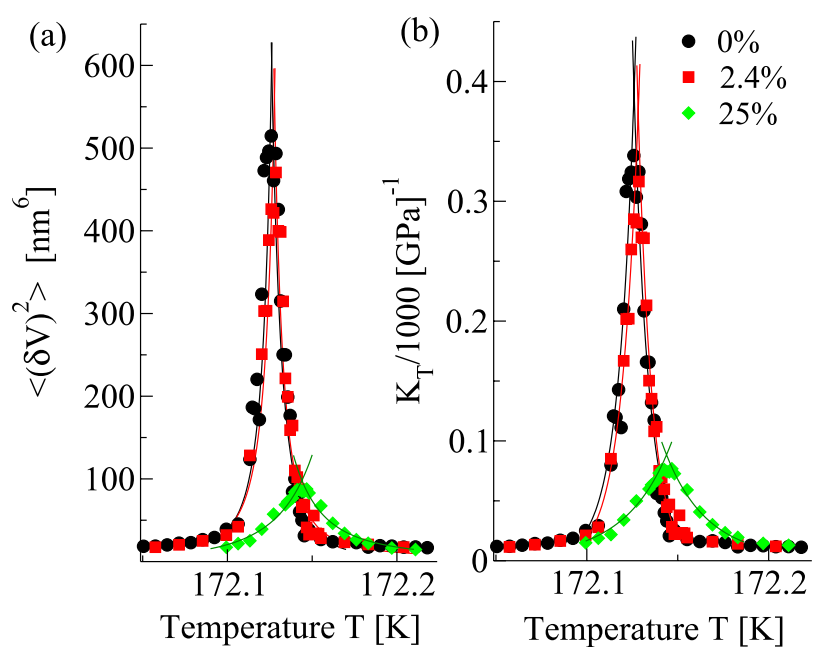

Figure 3. Decrease of volume fluctuations $\left\langle(\delta V)^{2}\right\rangle$ and isothermal compressibility $K_{T}$ at $P=0.14 \mathrm{GPa}$ for increasing hydrophobic nanoparticle concentrations $c$. Calculations based on a system with $\mathscr{N}=4 \times 10^{4}$. Lines are fits of simulation data for $\left\langle(\delta V)^{2}\right\rangle$ (a) and $K_{T}$ (b) with exponential function $y=a_{0} \mathrm{e}^{a_{1} /\left(a_{2}-x\right)}$ separately on each side of the maximum, excluding the points near the maximum. (We estimate the maximum of each dataset as the crossing of the fits on each side of the maximum, plus or minus the distance of the crossing point from the curve maximum.)

phase boundaries. Moreover, from the fluctuations it is possible to calculate the measurable quantities such as the isothermal compressibility, the isobaric specific heat or the isobaric expansion coefficient that characterize the macroscopic behavior of the system and are relevant in many technological applications. For example, from the calculations of the volume fluctuations $\left\langle(\delta V)^{2}\right\rangle \equiv\left\langle(V-\langle V\rangle)^{2}\right\rangle$ using the fluctuation-dissipation relation it is possible to calculate the isothermal compressibility, $K_{T} \equiv\left\langle(\delta V)^{2}\right\rangle /\left(k_{\mathrm{B}} T\langle V\rangle\right)$ (figure 3). From each dataset at constant $P$, using separate fits on each side of the set maxima, we extrapolate the maxima of compressibility $K_{T}^{\max }$, and the maxima in $\left\langle(\delta V)^{2}\right\rangle$.

By calculating the value of $K_{T}$ for state points sampled in the $P-T$ plane for $T<190 \mathrm{~K}$ (figure 4(a)) we identify the location and shape of the locus of maxima of $K_{T}$. When a critical point occurs, the locus of $K_{T}^{\max }$ calculated in the supercritical region converges towards the locus of the maxima of correlation length $\xi$ (Widom line), as well as any other locus of maxima of response functions. This is because in the vicinity of a critical point all the response functions can be expressed as a power law of $\xi$. On the other hand, at a critical point, $\xi$ diverges in the thermodynamic limit. Therefore, $K_{T}^{\max }$ and all the other response functions diverge at the critical point. Hence, by following the locus $K_{T}^{\max }(P)$ in the $P-T$ plane, when a critical point occurs we observe a diverging $K_{T}^{\max }$ in the thermodynamic limit. Moreover, because the critical point is at the end of a first-order phase transition in the $P-T$ plane, maxima occur along this line and increase linearly with system size. Therefore, by locating the locus of $K_{T}^{\max }$ and calculating the finite size scaling of $K_{T}^{\max }$ at different pressures we are able to locate the critical point and the line of the first-order phase transition. 

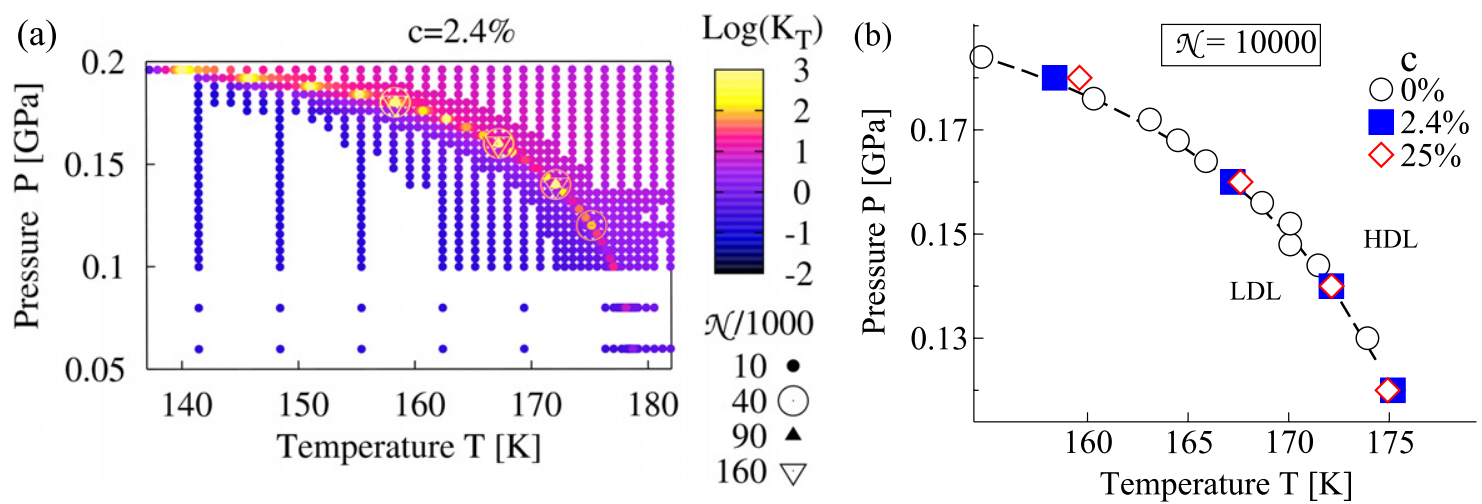

Figure 4. (a) The locus of maxima of $K_{T}(P, T)$ does not depend on $\mathscr{N}$, as shown by symbols for $\mathscr{N}$ going from $10^{4}$ to $1.6 \times 10^{5}$, for hydrophobic nanoparticles with radius $R=1.6 \mathrm{~nm}$ and $c=2.4 \%$. Values of $\log K_{T}(P, T)$ are color-coded as shown in the panel. (b) The locus of maxima of $K_{T}(P, T)$ does not depend either on concentrations $c$, as shown for $\mathscr{N}=10^{4}$ and $c=0,2.4 \%$ and $25 \%$.
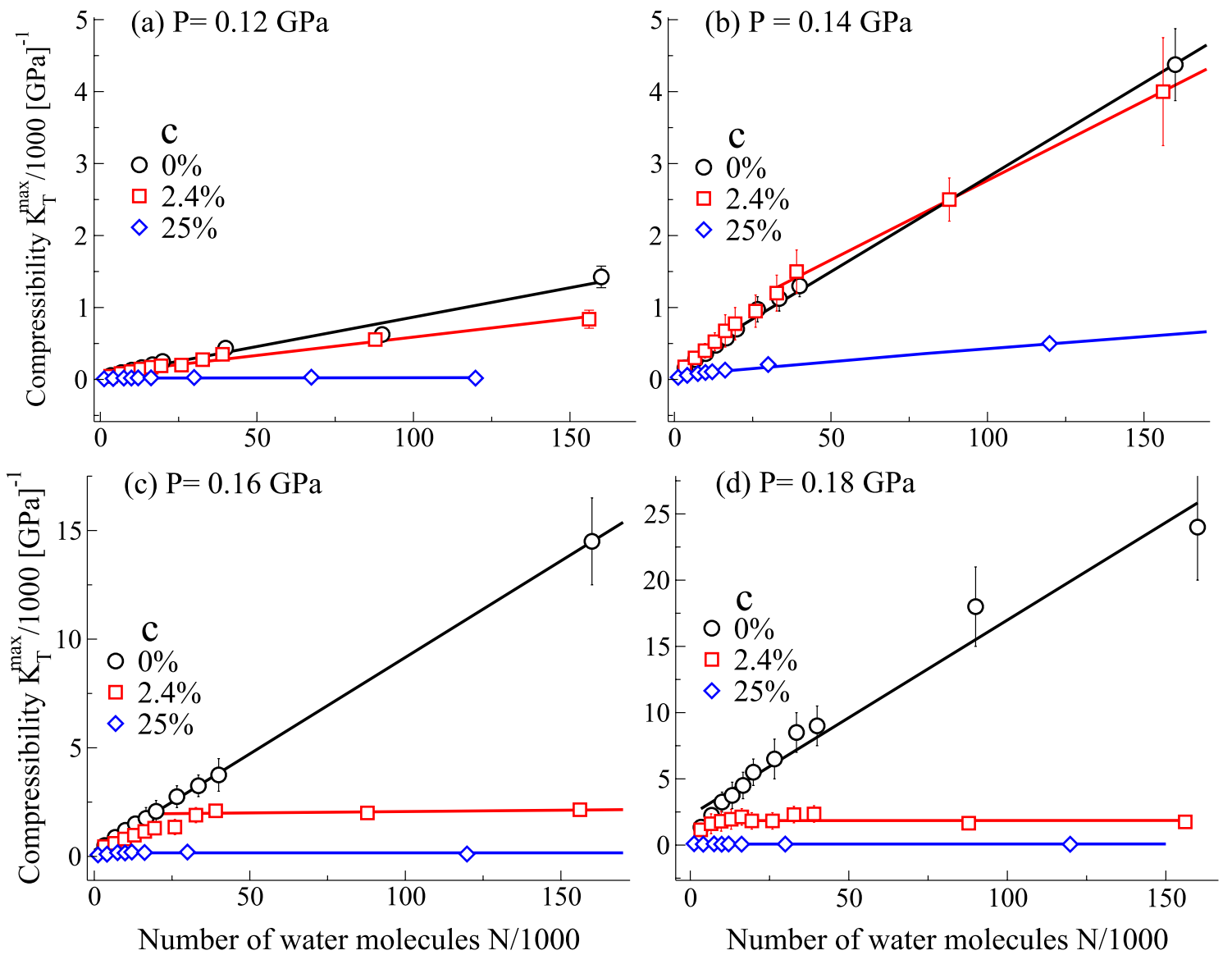

Figure 5. Dependence of the maxima $K_{T}^{\mathrm{max}}$ of the isothermal compressibility $K_{T} \equiv\left\langle(\delta V)^{2}\right\rangle /\left(k_{\mathrm{B}} T\langle V\rangle\right)$ for $c=0,2.4 \%$ and $25 \%$ on the number of water molecules $N$. (a) For $P=0.12 \mathrm{GPa}, K_{T}^{\max }$ slowly increases and possibly saturates. (b) For $P=0.14 \mathrm{GPa}$, the linear increase of $K_{T}^{\max }$ with $N$ is consistent with a first-order LLPT for all $c$. For $P=0.16 \mathrm{GPa}$ (c) and $P=0.18 \mathrm{GPa}$ (d), $K_{T}^{\mathrm{max}}$ increases linearly only for $c=0$, indicating a first-order LLPT, but saturates for $c=2.4 \%$ and $25 \%$, consistent with the absence of a first-order LLPT ${ }^{4}$.

We first extract the locus of maxima of $K_{T}$ at different values of $c$ and observe that its position does not depend on $\mathscr{N}$ (figure 4(a)) nor $c$ (figure 4(b)). We therefore conclude that

4 At $c=0, K_{T}^{\max }$ increases for higher $P$ because $\left\langle(\delta V)^{2}\right\rangle$ depends weakly on $P$, and $K_{T}^{\max }$ occurs at lower $T\langle V\rangle$. the line of $K_{T}^{\max }$ is a robust feature of water upon confinement in a fixed disordered hydrophobic matrix of hydrophobic nanoparticles.

We find, however, that confinement drastically reduces volume fluctuations at low $T$. For a water monolayer with $\mathscr{N}=1.6 \times 10^{5}$ cells confined within hydrophobic 


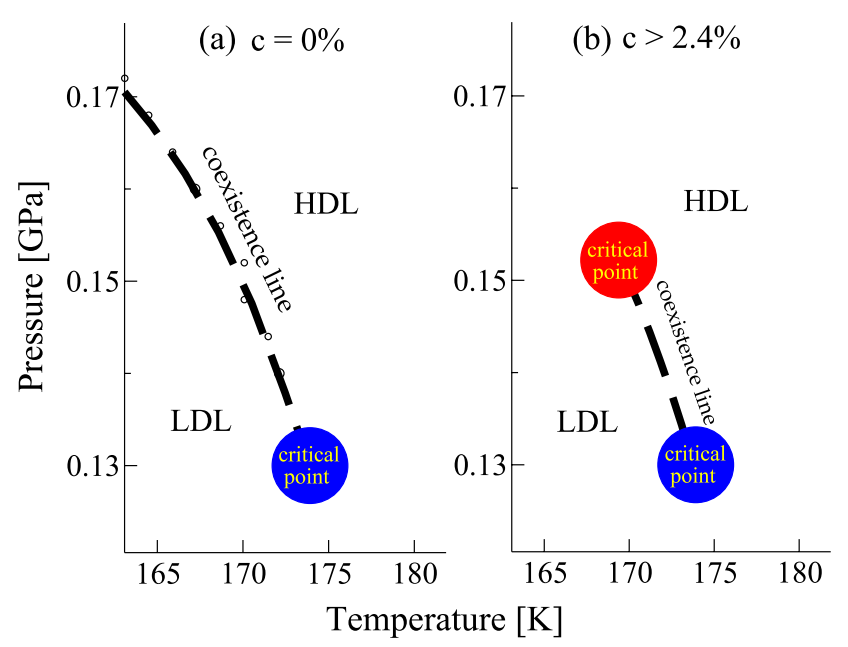

Figure 6. Enlarged view of the low- $T$ region of the phase diagram. The first-order LLPT ends in a critical point at $T \simeq 174 \mathrm{~K}$ and $P \simeq 0.13 \mathrm{GPa}$ for all $c$. In (a) for $c=0$ the first-order LLPT is terminated by one critical point. For $c=2.4 \%$ and $25 \%$ (b) at $P>0.15 \mathrm{GPa}$ the first-order LLPT is no longer detected, indicating a new high- $P$ end point, whose behavior is consistent with a critical point (see text).

nanoparticles with $R=1.6 \mathrm{~nm}$ at $c=25 \%$, we find a maximum $K_{T}^{\max }$ along the isobar at $P \simeq 0.16 \mathrm{GPa}$ that is $99.7 \%$ smaller than the $c=0$ case. If we decrease $c$ to $2.4 \%$, the reduction of $K_{T}^{\max }$ is still remarkable: $92.3 \%$ (figure 5).

For $c=0$, previous investigations have located a liquid-liquid critical point at $T \simeq 174 \mathrm{~K}$ and $P \simeq 0.13 \mathrm{GPa}$ by calculating the fluctuations of volume and entropy (figure 6(a)) [34-40].

Note that for $c>0$ the maxima of $\left\langle(\delta V)^{2}\right\rangle$ do not change monotonically with $P$ or $T$ (figure 7). Instead, the maxima of $\left\langle(\delta V)^{2}\right\rangle$ appear to diverge at two different values of $T$ and $P$. This behavior for $c>0$ is consistent with the occurrence of another critical point at high $P$ (figure $6(\mathrm{~b})$ ).

The general theory of finite-size scaling tells us that, at a first-order phase transition, $K_{T}^{\max }$ increases linearly with the number of degrees of freedom, here equal to $4 N$. We find a clear linear increase for $0.14 \mathrm{GPa} \leq P \leq 0.20 \mathrm{GPa}$ for $c=0$, and only for $0.14 \mathrm{GPa} \leq P<0.16 \mathrm{GPa}$ for $c=25 \%$ and $2.4 \%$, consistent with the absence of a first-order LLPT outside these ranges (figure 5).

To emphasize that, at low $P, K_{T}^{\mathrm{max}}$ does not grow linearly with $N$, we consider the $\chi \equiv \log \left(K_{T}^{\max } \times 1 \mathrm{GPa} / N\right)$ as a function of $1 / N$, where $K_{T}^{\max } \times 1 \mathrm{GPa} / N$ is dimensionless (figure 8)

For a first-order LLPT $\chi$ tends to a constant as $1 / N \rightarrow$ 0 , otherwise it vanishes. Our calculations confirm that a first-order LLPT is absent for any $c$ at sufficiently low $P$. For $c=2.4 \%$ and $25 \%$ the first-order LLPT is absent also at sufficiently high $P$. Hence there is an upper critical point at the end of the LLPT line for $c=2.4 \%$ and $25 \%$.

To more precisely locate the end points of the first-order LLPT, we study the finite-size scaling of the Binder cumulant for volume [84, 85]

$$
U_{\mathscr{N}} \equiv 1-\frac{\left\langle V^{4}\right\rangle_{\mathscr{N}}}{3\left\langle V^{2}\right\rangle_{\mathscr{N}}^{2}},
$$

where $\langle\cdot\rangle_{\mathscr{N}}$ is the thermodynamic average for a system with $\mathscr{N}$ cells. For $\mathscr{N} \rightarrow \infty$, at fixed $c$ and $P, U_{\mathscr{N}}=2 / 3$ for any $T$ away from a first-order phase transition, while $U_{\mathscr{N}}^{\min }<2 / 3$ at a first-order phase transition [84, 85].

The $T$ dependence of $U_{\mathscr{N}}^{\mathrm{min}}$ is shown in figures 9(a) and (b) for $c=0$ at two representative values of $P$ and for different system sizes. We find that there is a temperature at which $U_{\mathscr{N}}^{\min }<2 / 3$ with increasing $\mathscr{N}$ for $P \geqslant 0.14 \mathrm{GPa}$, indicating the presence of a first-order LLPT in this region. The Binder cumulant analysis therefore confirms what we concluded from the behavior of $K_{T}^{\mathrm{max}}$, i.e. the existence of a first-order LLPT in the range $0.14 \mathrm{GPa} \leqslant P \leqslant 0.2 \mathrm{GPa}$ for $c=0$. However, for $c=2.4 \%$ and $25 \%$, we find that with increasing $\mathscr{N}$ there is a $T$ at which $U_{\mathscr{N}}^{\min }<2 / 3$ for $P=$ $0.14 \mathrm{GPa}$, but not for $P \geqslant 0.18 \mathrm{GPa}$ (figures 9 (c)-(f)), which implies that in the thermodynamic limit at $P=0.18 \mathrm{GPa}$ the system is in the one-phase region at all the temperatures considered here. These results are summarized for several pressures in figure 10, where we show the size dependence of
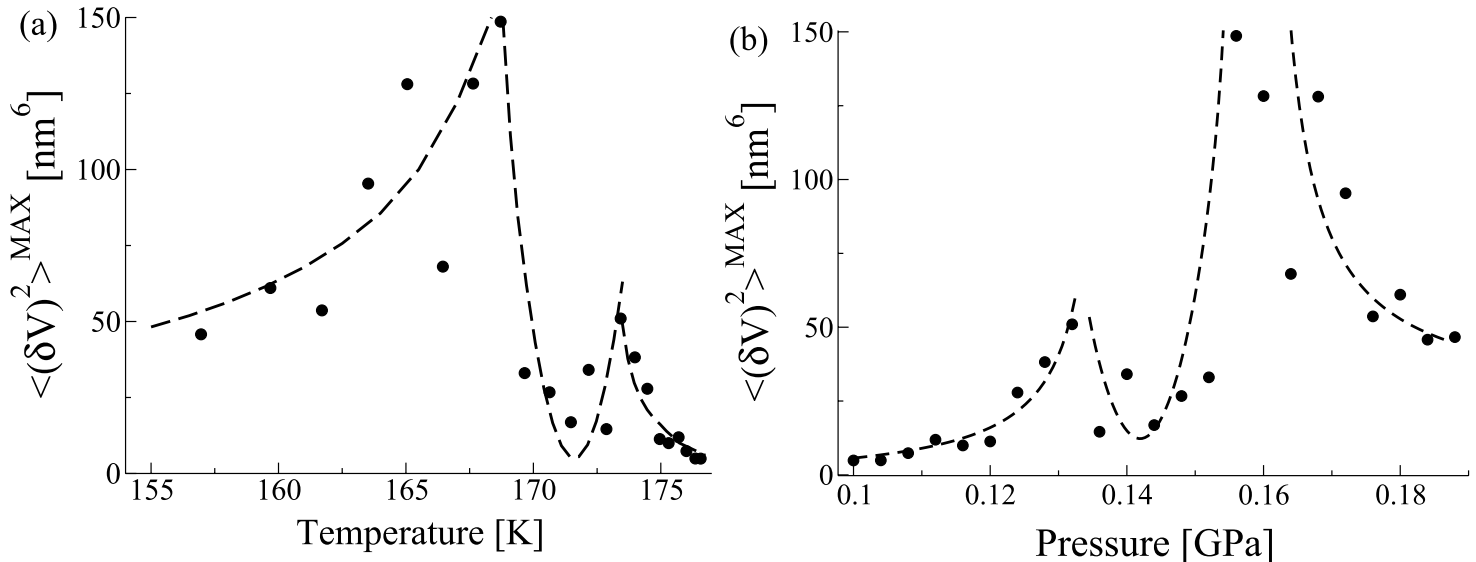

Figure 7. The maxima of $\left\langle(\delta V)^{2}\right\rangle$ for $c=25 \%$ and $\mathscr{N}=10^{4}$. (a) $\left\langle(\delta V)^{2}\right\rangle^{\text {max }}$ increase approaching $T=168$ and $174 \mathrm{~K}$ and (b) approaching $P=0.132$ and $0.156 \mathrm{GPa}$. Dashed lines are guides for the eyes. 


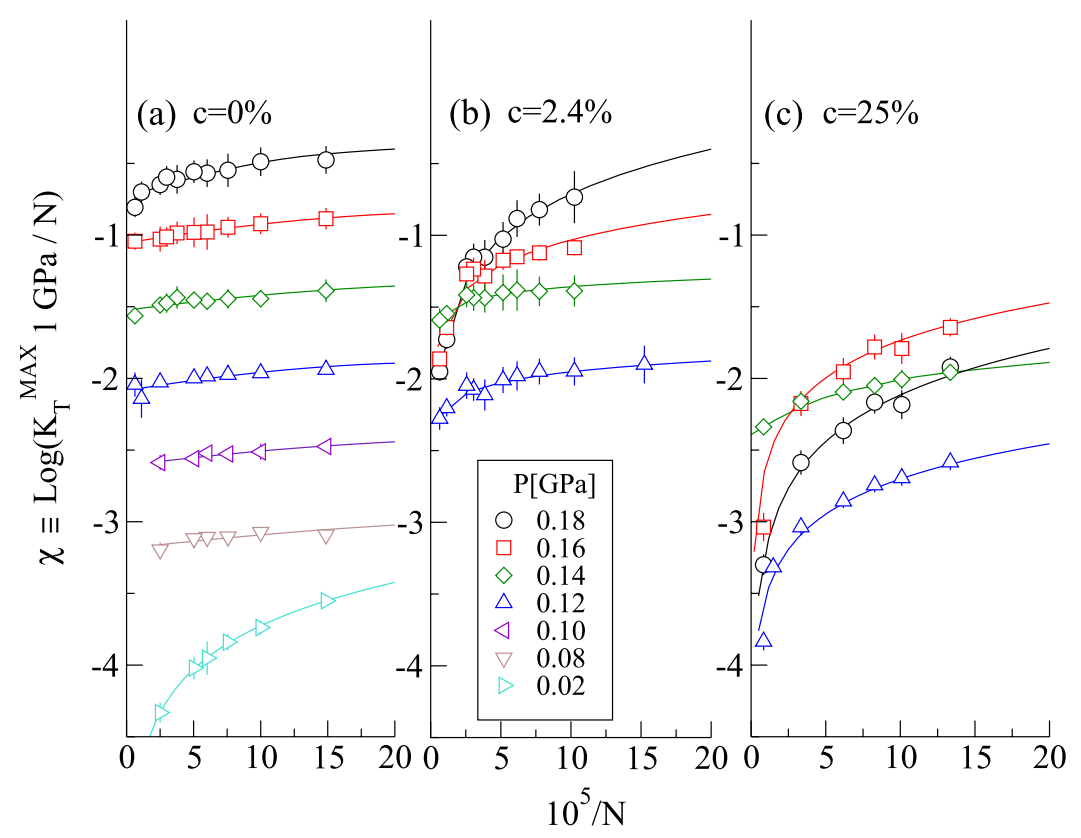

Figure 8. Size dependence of compressibility maxima $K_{T}^{\max }$ for several pressures and (a) $c=0$, (b) $c=2.4 \%$ and (c) $c=25 \%$. The dimensionless quantity $\chi \equiv \log \left(K_{T}^{\max } \times 1 \mathrm{GPa} / N\right)$ has a clear decrease for $c=0$ only for very low $P$, indicating the absence of a first-order LLPT. At fixed $P$, by increasing $c$, the value of $K_{T}^{\max } / N$ decreases of orders of magnitude. For $c=25 \%$ the values of $\chi$ are not monotonic with $P$, with maxima at $P=0.16 \mathrm{GPa}$, showing that the upper end point for $c=25 \%$ is between $P=0.16$ and $0.14 \mathrm{GPa}$.

the minima of the Binder cumulant at different $P$ for the three concentrations studied. For $c=2.4 \%$ and $25 \%$ we find that $U_{\mathscr{N}}^{\min }$ tends to a value less than $2 / 3$, consistent with a first-order LLPT only for $P$ at about $0.14 \mathrm{GPa}$. Therefore, consistent with what has already been indicated by the analysis of $K_{T}^{\max }$ and $\left\langle(\delta V)^{2}\right\rangle$ (figures 5 and 7 ) for $c=2.4 \%$ and $25 \%$, the first-order LLPT occurs only in a limited range of pressures around $0.14 \mathrm{GPa}$, with two end points: one at $\approx 0.15 \mathrm{GPa}$ and the other at $\approx 0.13 \mathrm{GPa}$ (figure 6(b)).

\section{Discussion}

We adopt density as the relevant order parameter for the liquid-liquid phase transition. In the model the density depends on the number of accessible configurations (entropy) and energy of the system via the number of $\mathrm{HBs} N_{\mathrm{HB}}$, and their specific volume $v_{\mathrm{HB}}$. Therefore, our order parameter includes by definition contributions coming from entropy and energy, as is, in general, the case for fluid-fluid phase transitions [86].

It is interesting to compare our results with other cases in which the effect of quenched disorder on phase transitions in 2D has been considered, including membranes [87-89] or quenched filler particles [90]. In particular, we are interested in the comparison with the case of random-field (RF) quenched disorder. In the RF case, phase transitions are always destroyed in two dimensions [91-93]. To clarify what type of quenched disorder do the hydrophobic nanoparticles represent in the case considered here, we observe the following. For RF quenched disorder two conditions must hold: (i) the RF couples with the order parameter and (ii) the $\mathrm{RF}$ destroys the ground state by frustrating it. While in our model the first condition (i) holds, the second one (ii) does not, as we discuss in the following. Hence, our system cannot be modeled as an RF.

The RF destroys the ground state, because it locally forces the degrees of freedom to assume states that are not consistent with the ground state. In other words, the RF forces the creation of interfaces. This is not true in our model, where the ground state is not modified by the presence of the hydrophobic nanoparticles. By starting from a completely ordered configuration (one of the $q$ ground states), we heat up the sample and observe that the stronger water-water hydrogen bond (HB) interactions near the hydrophobic nanoparticles make the ground state as stable as the case without the hydrophobic nanoparticles, as shown in figure 4 , where the locus of the maxima of $K_{T}(P, T)$ does not depend on concentration $c$.

We understand our results in terms of dilution-quenched disorder, for which the disorder effects are mild and do not destroy phase transitions in 2D. In particular, by including hydrophobic nanoparticles, we delete water-water interactions in the region occupied by the hydrophobic nanoparticles and make the water-water interaction stronger for the first shell of water molecules hydrating the hydrophobic nanoparticles. The procedure reminds us of the one performed in the Kasteleyn-Fortuin dilution that, as can be analytically demonstrated [82], does not change the thermodynamics of the Ising model or any Potts model. Thus we believe that our model cannot be described as an RF model and the results for RF models do not apply to our case.

However, in our case, at high $P$ the phase diagram changes. We believe that this effect could be a consequence of the fact that we do not follow the rules of Kasteleyn-Fortuin 

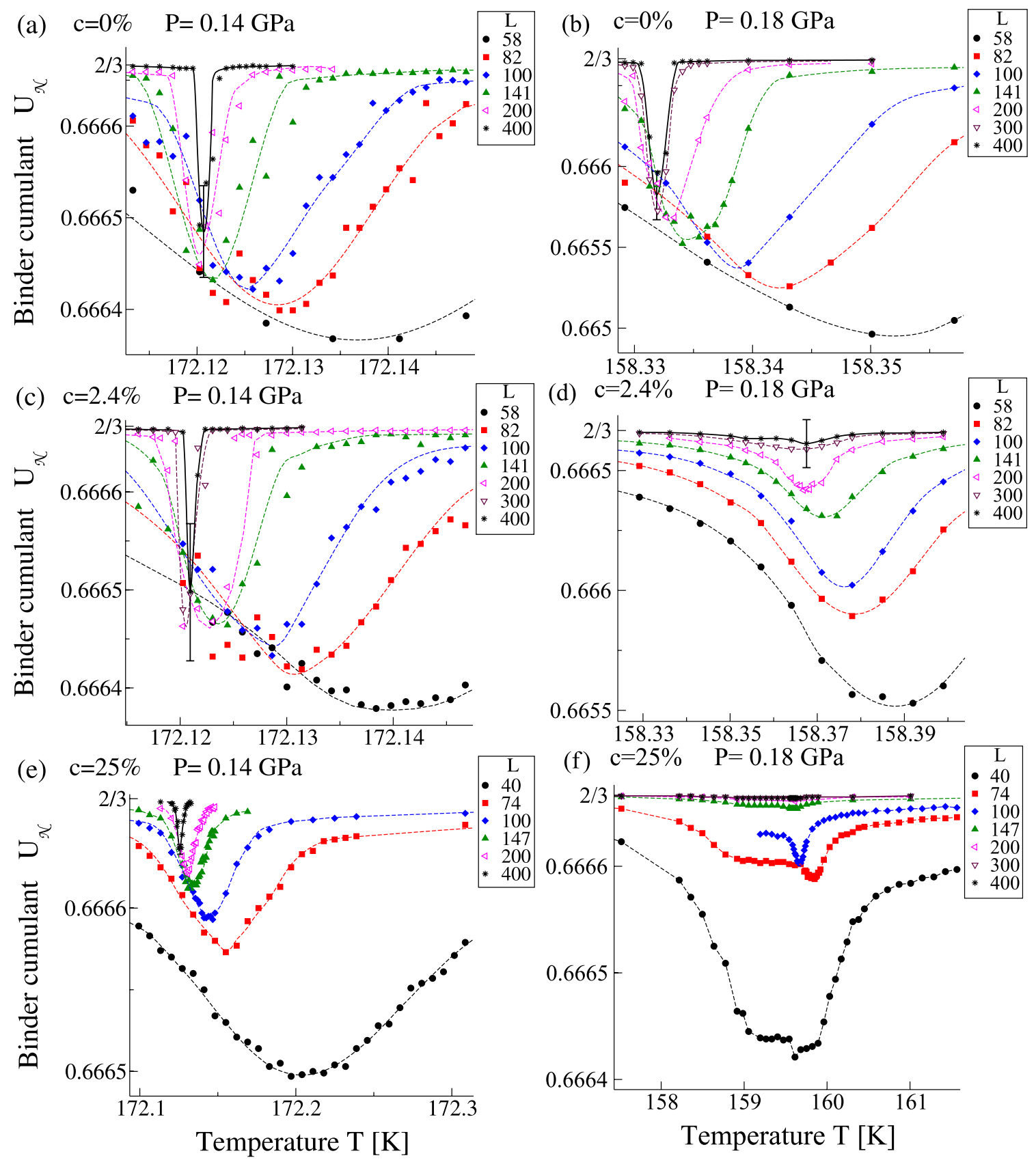

Figure 9. Finite-size scaling of the isobaric Binder cumulant $U_{\mathscr{N}}$ as a function of temperature, for different values of hydrophobic nanoparticle concentrations $c$. Sizes range from $\mathscr{N}=L^{2}=1600$ to $1.6 \times 10^{5}$. (a) For $c=0$ at pressure $P=0.14 \mathrm{GPa}$ and (b) $0.18 \mathrm{GPa}$; (c) for $c=2.4 \%$ at $P=0.14 \mathrm{GPa}$ and (d) $0.18 \mathrm{GPa}$; (e) for $c=25 \%$ at $P=0.14 \mathrm{GPa}$ and (f) $0.18 \mathrm{GPa}$. The error bars are estimated using the jackknife method for all the points, but only the largest error bars—at the minima of the largest system sizes-are shown for clarity reasons.

dilution, the only one that does not change the thermodynamics [83]. This consideration is supported by mean-field results for the model with no hydrophobic nanoparticles [37]. It has been shown [37] that the low- $T$ state is disordered around the locus $P_{\mathrm{LL}} \simeq\left(\left(J+3 J_{\sigma}\right) / v_{\mathrm{HB}}\right)+\gamma T_{\mathrm{LL}}$, where $T_{\mathrm{LL}}$ and $P_{\mathrm{LL}}$ are the $T$ and $P$ along the liquid-liquid transition line and $\gamma \simeq-7.4 k_{\mathrm{B}} / v_{0}$. The disordering is continuous at low $P$ and discontinuous at high $P$ with a critical point $\left(P_{\mathrm{C}}, T_{\mathrm{C}}\right)$, separating the low- $P$ and high- $P$ region, given by

$$
T_{\mathrm{C}} \simeq(4 / 3) J_{\sigma} / \epsilon
$$

and

$$
P_{\mathrm{C}} \simeq\left(\left(J+3 J_{\sigma}\right) / v_{\mathrm{HB}}\right)+\gamma T_{\mathrm{C}} .
$$

With the hydrophobic nanoparticles, we introduce new energy scales $J^{\mathrm{h}}=1.3 \mathrm{~J}$ and $J_{\sigma}^{\mathrm{h}}=1.3 J_{\sigma}$ with stronger HBs. We now expect that at high enough $P_{\mathrm{LL}}^{\prime} \simeq\left(\left(J^{\mathrm{h}}+3 J_{\sigma}^{\mathrm{h}}\right) / v_{\mathrm{HB}}\right)+$ $\gamma T_{\mathrm{LL}}^{\prime}=P_{\mathrm{LL}}+0.3\left(J+J_{\sigma}\right) / v_{\mathrm{HB}}>P_{\mathrm{C}}+0.3\left(J+J_{\sigma}\right) / v_{\mathrm{HB}}>P_{\mathrm{C}}$, with $T_{\mathrm{LL}}^{\prime} \simeq\left(J^{\mathrm{h}}+3 J_{\sigma}^{\mathrm{h}}-P_{\mathrm{LL}}^{\prime} v_{\mathrm{HB}}\right) /(-\gamma)=T_{\mathrm{LL}}-0.3(J+$ $\left.J_{\sigma}\right) /(-\gamma)<T_{\mathrm{C}}-0.3\left(J+J_{\sigma}\right) /(-\gamma)<T_{\mathrm{C}}$, both stronger HBs and normal HBs are weakened enough by the effect of 

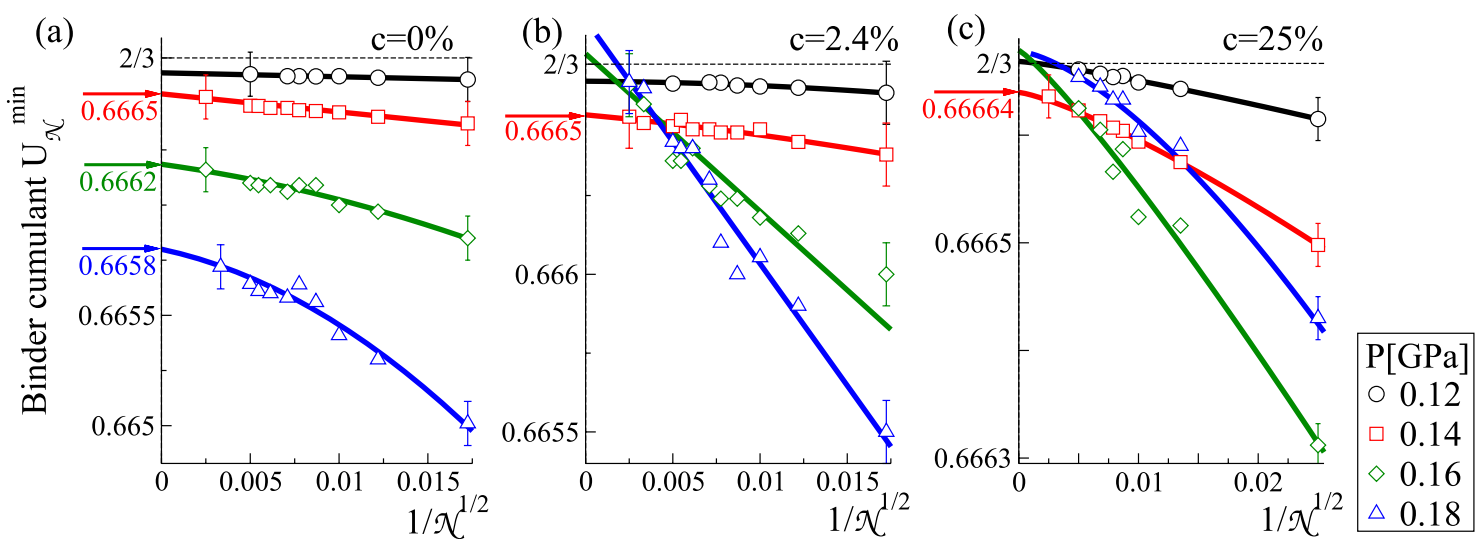

Figure 10. Extrapolation of the minima of Binder cumulants $U_{\mathscr{N}}^{\min }$ to the thermodynamic limit $\mathscr{N} \rightarrow \infty$. For $P=0.12 \mathrm{GPa}, U_{\mathscr{N}}^{\mathrm{min}} \rightarrow 2 / 3$ as $\mathscr{N} \rightarrow \infty$ within the error bars. For $c=0 \%$ in (a) $U_{\mathscr{N}}^{\min } \leqslant 2 / 3$ for $P \geqslant 0.14 \mathrm{GPa}$, indicating a first-order LLPT for these pressures. For hydrophobic nanoparticle concentrations of $c=2.4 \%$ (b) and $c=25 \%$ (c), $U_{\mathcal{N}}^{\min } \leqslant 2 / 3$ for $\mathscr{N} \rightarrow \infty$ only for $P=0.14$ GPa marked by the arrow. Therefore, the LLPT is washed out by the hydrophobic confinement at high $P$. For the sake of clarity, typical error bars are shown only for a few points. Lines through the points are polynomial fits.

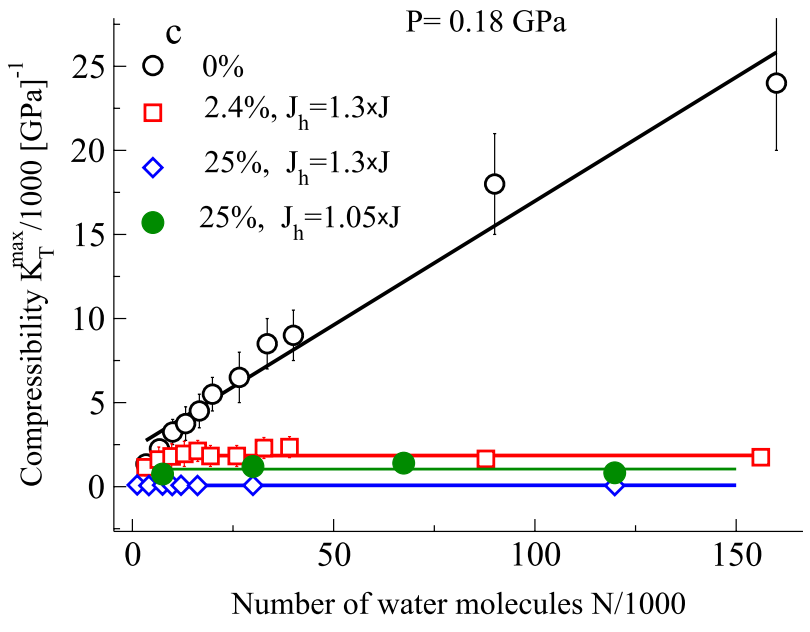

Figure 11. Dependence of the isothermal compressibility maxima $K_{T}^{\max }$ on the number of water molecules $N$ at pressure $P=0.18 \mathrm{GPa}$ for simulations of the model with $J^{\mathrm{h}}=1.3 \mathrm{~J}$ for $c=0,2.4 \%$ and $25 \%$ (open symbols), as in figure 5, and for the model with $J^{\mathrm{h}}=1.05 \mathrm{~J}$ for $c=25 \%$ (filled circles). We observe quantitative, but not qualitative, differences between the two cases with the same $c=25 \%$ and different $J^{\mathrm{h}}$.

$P$. The random distribution of distances between hydrophobic nanoparticles makes the disordering process continuous at $P>P_{\mathrm{LL}}^{\prime}$ and $T<T_{\mathrm{LL}}^{\prime}$. This interpretation seems to be qualitatively consistent with our numerical results.

It is also important to note that we choose not to perform averages over the randomness because our results show that the system is self-averaging for increasing size. Indeed, we change the random configuration of hydrophobic nanoparticles for each size that we consider and we find that the results converge to a limit for increasing size, as shown in figures 8-10. Therefore, since the system is self-averaging, averages over randomness are not going to change our results.

The relation of this model in $2 \mathrm{D}$ with bulk $3 \mathrm{D}$ water is established by the mean-field results for the model [37]. Mean-field applies to systems embedded in a space with large or infinite dimensions. It was shown that, apart from quantitative differences with simulations in $2 \mathrm{D}$, mean-field results and $2 \mathrm{D}$ results agree qualitatively. This agreement is strong evidence that the embedding dimension does not play a role in the qualitative predictions of the model. Furthermore, comparison of the phase diagram of the 2D model simulations with the results from 3D models do not show qualitative differences and, for several properties, not even quantitative differences, as discussed in [39].

We finally discuss here the effect of the increased $\mathrm{HB}$ strength for water-water interactions at the surface of the hydrophobic nanoparticles. The value $J^{\mathrm{h}}=1.3 \mathrm{~J}$ is chosen to facilitate the comparison with previous results from other models [80]. However, our preliminary results for $J^{\mathrm{h}}=1.05 \mathrm{~J}$ and $c=25 \%$ do not show qualitative differences with the case with $J^{\mathrm{h}}=1.3 \mathrm{~J}$ and the same concentration of hydrophobic nanoparticles (figure 11).

\section{Conclusion}

As a consequence of the restructuring effect of hydrophobic nanoparticles on the hydration shell water, stronger HBs are formed in the hydration shell of each solute. At low $T$ the hydration water is more ordered with respect to the $c=0$ case. However, hydration shells around different hydrophobic nanoparticles have a high probability $(5 / 6 \simeq 83 \%)$ of being in a different local bonding order. Consequently, different ordered domains are generated in the vicinity of hydrophobic nanoparticles, reminiscent of the locally structured regions proposed in [76]. These competing domains disrupt the macroscopic order (figure 12). Due to the presence of many domain boundaries there is a large decrease in the fluctuations and response functions, such as $K_{T}$. In contrast, when no hydrophobic nanoparticles are introduced into the system $(c=$ 0 ), a small temperature increase leads to a rapid change from the ordered to a disordered configuration as expected for a first-order phase transition (figure 13). This drastic change 


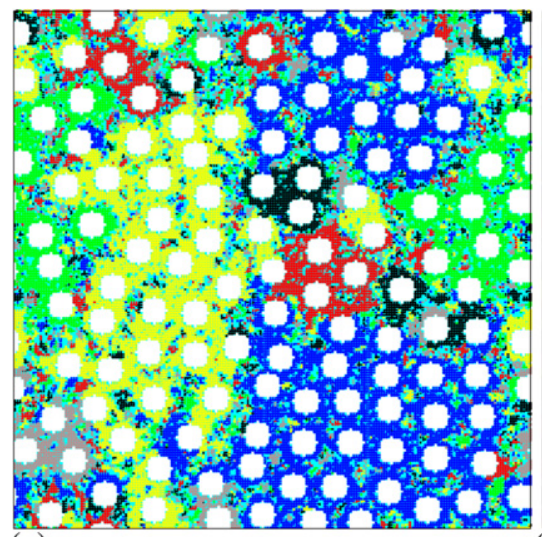

(a)

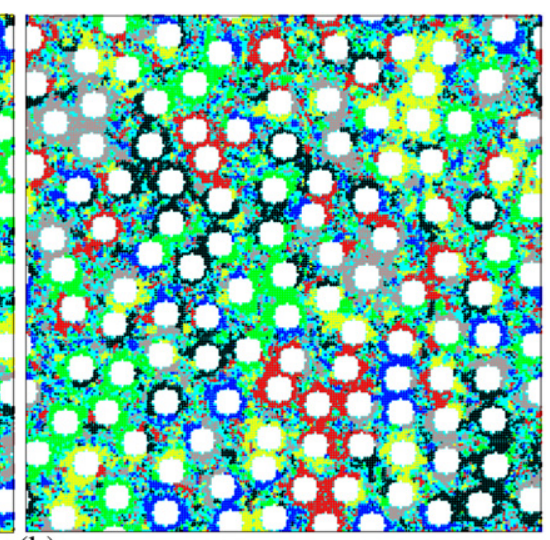

(b)

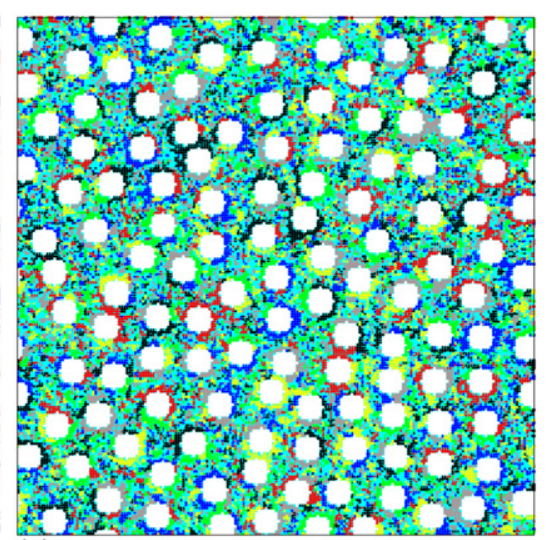

(c)

Figure 12. Typical configurations for $c=25 \%$ and $P=0.18 \mathrm{GPa}$ for (a) $T=159.58 \mathrm{~K}$, (b) $160.31 \mathrm{~K}$ and $162.40 \mathrm{~K}$. Different colors represent different ordered domains of HBs. Water molecules with fewer than four HBs are represented in turquoise and hydrophobic nanoparticles in white. HB ordering is favored around hydrophobic nanoparticles, but the system is macroscopically disordered. Note that domain boundaries can cross the hydrophobic nanoparticles.
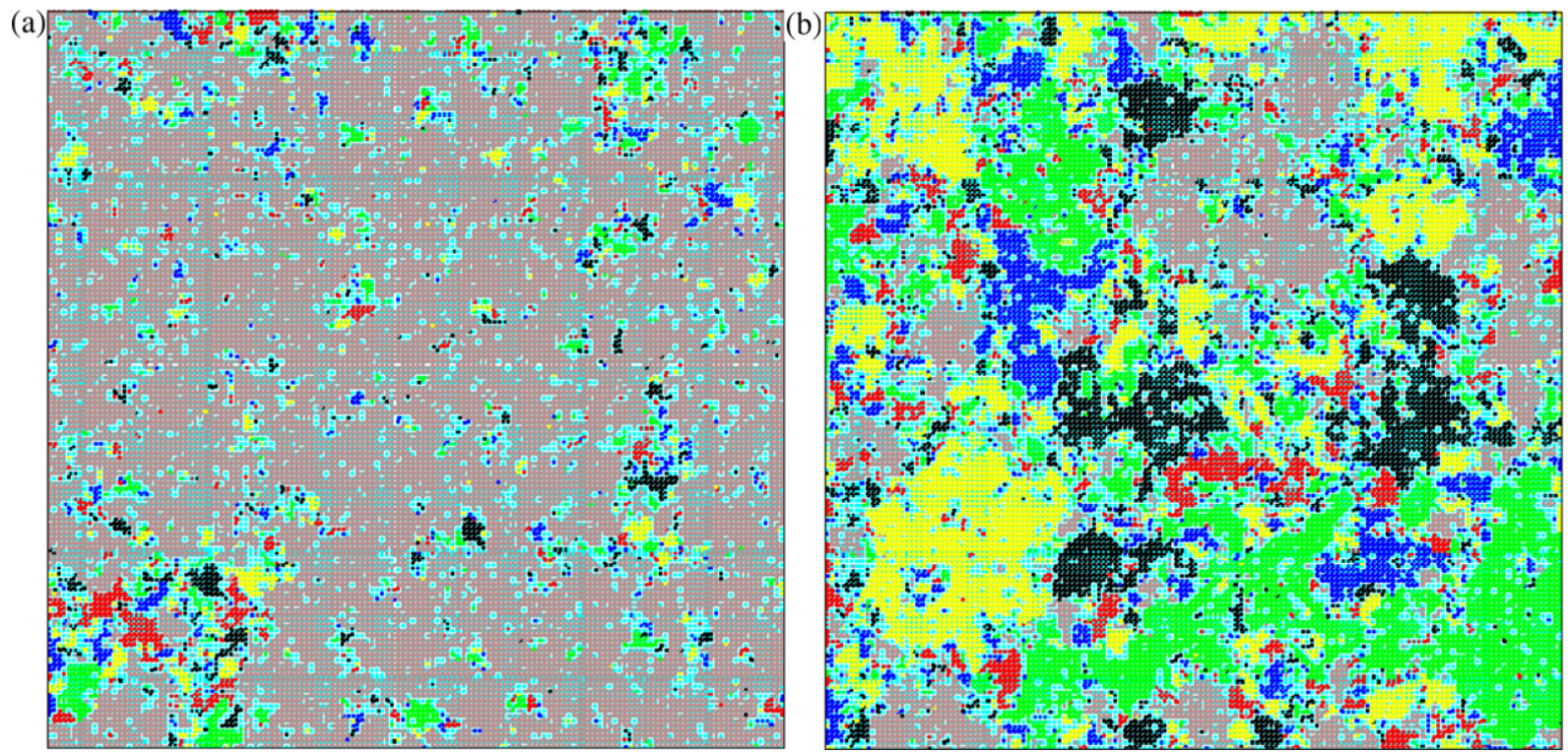

Figure 13. For $c=0$ a small temperature increase from (a) $T=158.332 \mathrm{~K}$ to (b) $158.333 \mathrm{~K}$ leads to a sudden change from the ordered (a) to a disordered configuration (b). Such behavior is consistent with a first-order phase transition.

is the origin of the large fluctuations in $K_{T}$ observed when approaching the discontinuous transition (figure 5).

Our results for $c=25 \%$ and $2.4 \%$ (figure 14) show that the smaller the $c$, the larger the $N$ at which the behavior deviates from the case $c=0 \%$. This observation suggests that the decrease of the fluctuations is due to the introduction of a characteristic length scale, inversely proportional to $c$, that limits the growth of the ordered structured regions. This is consistent also with a visual inspection of the typical configuration at different values of $c$ (figures 12-14).

Note that the reduction of compressibility was previously observed in the theoretical analysis of water confined by a fixed matrix of randomly distributed Lennard-Jones discs. However this reduction only occurred when the hydrophobic obstacle concentrations were high [94]. We instead find here that $K_{T}$ is reduced for $c$ as low as $2.4 \%$. More systematic studies are needed to understand how this difference among theoretical predictions depends on the details of the hydrophobic-nanoparticle-water interaction energy. For example, we have shown here that a $30 \%$ increase in the HB strength in the water first hydration shell is enough to decrease the compressibility by $90 \%$ even for small $c$.

Our results are qualitatively consistent with recent experiments on $\mathrm{H}_{2} \mathrm{O}$ confined in the hydrophobic mesoporous material CMK-1-14 consisting of micrometer-sized grains, each with a three-dimensional interconnected bicontinuous pore structure, with an average pore diameter $14 \AA$, at a hydration level of $99 \%$ at ambient pressure [95]. Zhang et al find a broadening of the $\alpha_{\mathrm{P}}$ peak, spanning from 240 to $180 \mathrm{~K}$ in mesoporous CMK, in contrast to the sharp peak at $230 \mathrm{~K}$ in hydrophilic confinement in silica mesopores MCM [95], reminiscent of our results on the reduction of the response 


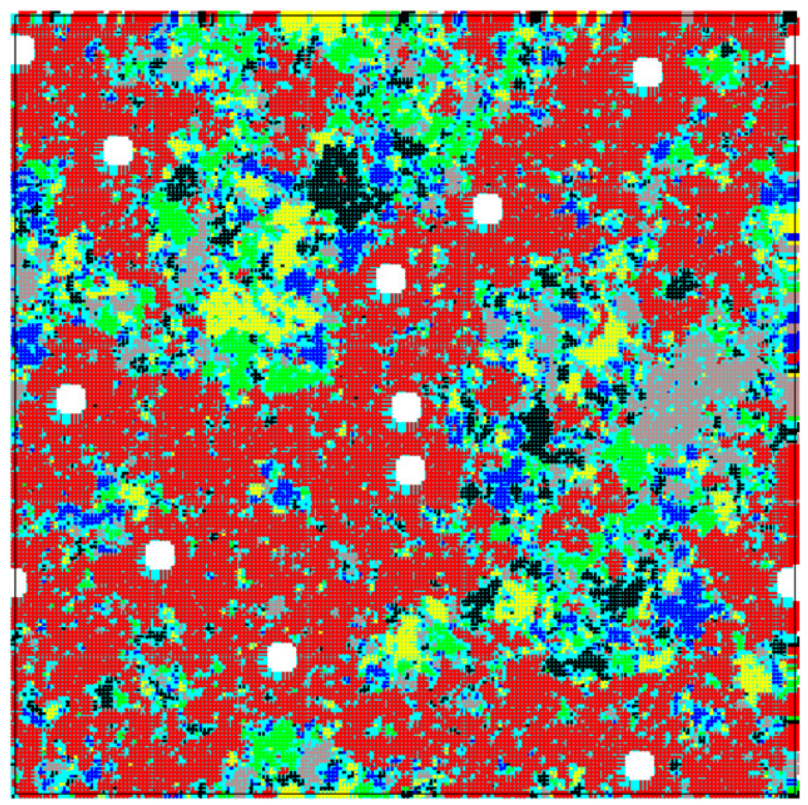

Figure 14. Configuration for $c=2.4 \%$ for $T=158.36 \mathrm{~K}$. The hydrophobic nanoparticles contribute to the continuous growth of the ordered domains, where the largest ordered domain is visualized in red.

functions with respect to the $c=0$ case. In addition, Zhang et al demonstrate the TMD downshift by $17 \mathrm{~K}$ in hydrophobic CMK with respect to the hydrophilic MCM, which is similar to our observed temperature downshift of TMD for low pressures.

Recent results for small-angle $\mathrm{x}$-ray scattering for aqueous solutions of amphiphilic tetraalkyl-ammonium at ambient conditions suggest that the strengthening of the structure of hydration water is present only for solutes with radius smaller than $\approx 0.44 \mathrm{~nm}$ [96]. We therefore repeat our analysis for small hydrophobic nanoparticles with $R=$ $0.4 \mathrm{~nm}$ and find that our results are robust if the amount of hydrophobic interface in contact with water is kept constant with respect to the case of $R=1.6 \mathrm{~nm}$. We conclude that the observed thermodynamic shifts and reductions of the response functions are due to the water-hydrophobic-nanoparticle interactions. In particular, they are directly related to the strength of the HBs in the hydration shell. As a consequence, by keeping a constant amount of water molecules in direct contact with the hydrophobic nanoparticles, we reproduce consistent shifts in spinodal, TMD and high- $P$, and the disappearance of the LLPT for both $R=1.6$ and $0.4 \mathrm{~nm}$ hydrophobic nanoparticles.

It was previously shown for $c=0$ that the dynamics of the Wolff cluster algorithm are very efficient and allow the system to fully equilibrate even in a low temperature region [73]. The dynamics of the $c>0$ systems are still under detailed investigation. However, our preliminary results demonstrate that introducing a hydrophobic confinement with restructuring effect does not slow down the equilibration process. At the present time more detailed study of the correlation times is needed.
In conclusion, we predict that a water monolayer confined in a hydrophobic fixed matrix of hydrophobic nanoparticles at concentration $c$ displays significant changes in the thermodynamics and important reductions in the response functions with respect to the $c=0$ case. Moreover, at $c$ as small as $2.4 \%$ a first-order LLPT at high $P$ is no longer detected. As a consequence, even a small number of hydrophobic nanoparticles can make the detection of the LLPT difficult. These findings may have important applications in fields related to conservation at cryogenic temperatures (around $-100^{\circ} \mathrm{C}$ ), for example, in the preservation of stem cells, blood or food products.

\section{Acknowledgments}

We thank S V Buldyrev, P Ch Ivanov and K Stokely for useful discussions and NSF grants CHE0908218 and CHE0911389, and MICINN grant FIS2009-10210 (FEDER) and the European Union FP7 grant NMP4-SL-2011-266737 for support.

\section{References}

[1] Mallamace F, Branca C, Corsaro C, Leone N, Spooren J, Chen S-H and Stanley H E 2010 Proc. Natl Acad. Sci. 10722457

[2] Franzese G, Hernando-Martinez A, Kumar P, Mazza M G, Stokely K, Strekalova E G, de los Santos F and Stanley H E 2010 J. Phys.: Condens. Matter 22284103

[3] Chen S-H and Loong C-K 2006 Nucl. Eng. Technol. 38201

[4] Bellissent-Funel M-C, Chen S H and Zanotti J-M 1995 Phys. Rev. E 514558

[5] Swenson J, Jansson H and Bergman R 2006 Phys. Rev. Lett. 96247802

[6] Mallamace F, Broccio M, Corsaro C, Faraone A, Wanderlingh U, Liu L, Mou C-Y and Chen S H 2006 J. Chem. Phys. 124161102

[7] Chen S-H, Mallamace F, Mou C-Y, Broccio M, Corsaro C and Faraone A 2006 Proc. Natl Acad. Sci. USA 10312974

[8] Liu D, Zhang Y, Chen C-C, Mou C-Y, Poole P-H and Chen S-H 2007 Proc. Natl Acad. Sci. USA 1049570

[9] Findenegg G H, Jähnert S, Akcakayiran D and Schreiber A 2008 ChemPhysChem 92651

[10] Angell C A 2008 Science 319582

[11] Mancinelli R, Bruni F and Ricci M A 2010 J. Phys. Chem. Lett. 11277

[12] Lervik A, Bresme F, Kjelstrup S, Bedeaux D and Rubi J M 2010 Phys. Chem. Chem. Phys. 121610

[13] Lervik A, Bresme F and Kjelstrup S 2009 Soft Matter 52407

[14] Bresme F, Chacón E, Tarazona P and Tay K 2008 Phys. Rev. Lett. 101056102

[15] Bresme F, Chacón E and Tarazona P 2008 Phys. Chem. Chem. Phys. 104704

[16] Ball P 2008 Chem. Rev. 10874

[17] Granick S and Bae S C 2008 Science 3221477

[18] Liu D, Zhang Y, Liu Y, Wu J, Chen C-C, Mou C-Y and Chen S-H 2008 J. Phys. Chem. B 1124309

[19] Mallamace F, Broccio M, Corsaro C, Faraone A, Liu L, Mou C-Y and Chen S-H 2006 J. Phys.: Condens. Matter $18 \mathrm{~S} 2285$

[20] Mallamace F, Broccio M, Corsaro C, Faraone A, Majolino D, Venuti V, Liu L, Mou C-Y and Chen S-H 2007 Proc. Natl Acad. Sci. USA 104424

[21] Han S, Kumar P and Stanley H E 2008 Phys. Rev. E 77 030201(R) 
[22] Giovambattista N, Rossky P J and Debenedetti P 2006 Phys. Rev. E 73041604

[23] Giovambattista N, Debenedetti P and Rossky P J 2007 J. Phys. Chem. B 1119581

[24] Majumder M, Chopra N, Andrews R and Hinds B J 2005 Nature 43844

[25] Joseph S and Aluru N R 2008 Phys. Rev. Lett. 101064502

[26] Gallo P, Rovere M and Chen S-H 2010 J. Phys. Chem. Lett. 1729

[27] Mishima O, Calvert L D and Whalley E 1984 Nature 310393

[28] Mishima O, Calvert L D and Whalley E 1985 Nature 31476

[29] Mishima O and Stanley H E 1998 Nature 396329

[30] Poole P H, Sciortino F, Essmann U and Stanley H E 1992 Nature 360324

[31] Poole P H, Essmann U, Sciortino F and Stanley H E 1993 Phys. Rev. E 484605

Harrington S, Poole P H, Sciortino F and Stanley H E 1997 J. Chem. Phys. 1077443

[32] Mishima O and Stanley H E 1998 Nature 392164

[33] Mishima O 2010 J. Chem. Phys. 133144503

[34] Franzese G and Stanley H E 2002 J. Phys.: Condens. Matter 142201

Franzese G and Stanley H E 2007 J. Phys.: Condens. Matter 19205126

[35] Franzese G, Marqués M I and Stanley H E 2003 Phys. Rev. E 67011103

[36] Kumar P, Franzese G and Stanley H E 2008 Phys. Rev. Lett. 100105701

[37] Stokely K, Mazza M G, Stanley H E and Franzese G 2010 Proc. Natl Acad. Sci. USA 1071301

[38] Mazza M G, Stokely K, Pagnotta S E, Bruni F, Stanley H E and Franzese G 2011 Proc. Natl Acad. Sci. USA 10819873

Kumar P, Yan Z, Xu L, Mazza M G, Buldyrev S V, Chen S H, Sastry S and Stanley H E 2006 Phys. Rev. Lett. 97177802

Sciortino F, Poole P, Stanley H E and Havlin S 1990 Phys. Rev. Lett. 64 1686-9

[39] de los Santos F and Franzese G 2011 J. Phys. Chem. B 11514311

[40] Franzese G, Stokely K, Chu X-Q, Kumar P, Mazza M G, Chen S-H and Stanley H E 2008 J. Phys.: Condens. Matter 20494210

[41] Paschek D 2004 J. Chem. Phys. 12010605

[42] Poole P H, Saika-Voivod I and Sciortino F 2005 J. Phys.: Condens. Matter 17 L431

[43] Liu Y, Panagiotopoulos A Z and Debenedetti P G 2009 J. Chem. Phys. 131104508

[44] Abascal J L F and Vega C 2005 J. Chem. Phys. 123234505

[45] Xu L, Kumar P, Buldyrev S V, Chen S-H, Poole P H, Sciortino F and Stanley H E 2005 Proc. Natl Acad. Sci. USA 10216558

[46] Limmer D T and Chandler D 2011 J. Chem. Phys. 135134503

[47] Poole P H, Becker S R, Sciortino F and Starr F W 2011 J. Phys. Chem. B 11514176

[48] Sciortino F, Saika-Voivod I and Poole P 2011 Phys. Chem. Chem. Phys. 1319759

[49] Kesselring T A, Franzese G, Buldyrev S V, Herrmann H J and Stanley H E 2011 Nanoscale dynamics of phase flipping in water near its hypothesized liquid-liquid critical point arXiv:1112.2186

[50] Debenedetti P G 2011 private communication

[51] Gallo P and Rovere M 2007 Phys. Rev. E 76061202

[52] Corradini D, Gallo P and Rovere M 2008 J. Chem. Phys. 128244508

[53] Kurita R and Tanaka H 2007 Phys. Rev. Lett. 98235701

[54] De Sanctis Lucentini P G and Pellicane G 2008 Phys. Rev. Lett. 101246101
[55] Fisher M E and Nakanishi H 1981 J. Chem. Phys. 755857

[56] Soper A K 2008 Mol. Phys. 1062053

[57] Ricci M A, Bruni F and Giuliani A 2009 Faraday Discuss. 141347

[58] Koga K, Tanaka H and Zeng X C 2000 Nature 408564

[59] Giovambattista N, Lopez C F, Rossky P J and Debenedetti P G 2008 Proc. Natl Acad. Sci. USA 1052274

[60] Franzese G and de los Santos F 2009 J. Phys.: Condens. Matter 21504107

[61] Truskett T M and Debenedetti P G 2001 J. Chem. Phys. 1142401

[62] Kumar P, Buldyrev S V, Starr F W, Giovambattista N and Stanley H E 2005 Phys. Rev. E 72051503

Starr F W, Nielsen J K and Stanley H E 1999 Phys. Rev. Lett. 822294

[63] Sastry S, Debenedetti P, Sciortino F and Stanley H E 1996 Phys. Rev. E 536144

Stanley H E 1979 J. Phys. A: Math Gen. 12 L329

[64] Franzese G and Stanley H E 2002 Physica A 314508

[65] Yesylevskyy S O, Schäfer L V, Sengupta D and Marrink S J 2010 PLoS Comput. Biol. 6 e 1000810

[66] Strekalova E G, Mazza M G, Stanley H E and Franzese G 2011 Phys. Rev Lett. 106145701

[67] Kumar P, Starr F W, Buldyrev S V and Stanley H E 2007 Phys. Rev. E 75011202

[68] Zangi R and Mark A E 2003 Phys. Rev. Lett. 91025502

[69] Pendás A M, Blanco M A and Francisco E 2006 J. Chem. Phys. 125184112

[70] Israelachvili J and Pashley R 1982 Nature 300341

[71] Debenedetti P G 2003 J. Phys.: Condens. Matter 15 R1669

[72] Soper A K and Ricci M A 2000 Phys. Rev. Lett. 842881

[73] Mazza M G, Stokely K, Strekalova E G, Stanley H E and Franzese G 2009 Comput. Phys. Commun. 180497

[74] Cicero G, Grossman J C, Schwegler E, Gygi F and Galli G 2008 J. Am. Chem. Soc. 1301871

[75] Doster W and Gutberlet T 2010 Biochim. Biophys. Acta 18043

[76] Frank H S and Evans M W 1945 J. Chem. Phys. 13507

[77] Stillinger F H 1973 J. Solut. Chem. 2141

[78] Chandler D 2005 Nature 437640

[79] Muller N 1990 Acc. Chem. Res. 2323

[80] Patel B A, Debenedetti P G, Stillinger F H and Rossky P J 2007 Biophys. J. 934116

[81] Lee B and Graziano G 1996 J. Am. Chem. Soc. 1185163

[82] Coniglio A and Peruggi F 1982 J. Phys. A: Math Gen. 151873

[83] Cataudella V, Franzese G, Nicodemi M, Scala A and Coniglio A 1996 Phys. Rev. E 54175

[84] Binder K 1981 Phys. Rev. Lett. 47693

[85] Vollmayr K, Reger J D, Scheucher M and Binder K 1993 Z. Phys. B 91113

[86] Bertrand C E and Anisimov M A 2011 J. Phys. Chem. B 11514099

[87] Machta B B, Papanikolaou S, Sethna J P and Veatch S L 2011 Biophys. J. 1001668

[88] Ehrig J, Petrov E P and Schwille P 2011 New J. Phys. 13045019

[89] Fischer T and Vink R L C 2011 J. Chem. Phys. 134055106

[90] Laradji M and MacNevin G 2003 J. Chem. Phys. 1192275

[91] Imry Y and Ma S K 1975 Phys. Rev. Lett. 351399

[92] Imry Y and Wortis M 1979 Phys. Rev. B 193580

[93] Aizenman M and Wehr J 1989 Phys. Rev. Lett. 62 2503s

[94] Urbic T, Vlachy V, Pizio O and Dill K A 2004 J. Mol. Liq. 11271

[95] Zhang Y, Liu K-H, Lagi M, Liu D, Littrell K C, Mou C-Y and Chen S-H 2009 J. Phys. Chem. B 1135007

Franzese G and Coniglio A 1998 Phys. Rev. E 582753

[96] Huang N, Nordlung D, Huang C, Tyliszczak T, Weiss T M, Acremann Y, Pettersson L G M and Nilsson A 2012 J. Chem. Phys. submitted 